\title{
Emprendimiento de base tecnológica: un análisis basado en economías innovadoras y eficientes
}

\section{Technology-based entrepreneurship: an analysis based on innovative and efficient economies}

URL: http://revistas.uta.edu.ec/erevista/index.php/bcoyu/article/view/883

DOI: http://dx.doi.org/10.31164/bcoyu.24.2020.883

\author{
Alex Aucanshala - Pilatuña ${ }^{1}$; Oscar Toainga - Curay²; Patricio Carvajal - Larenas ${ }^{3}$
}

Fecha de recepción: 13 de noviembre de 2019

Fecha de aceptación: 8 de enero de 2020

\section{Resumen}

El presente trabajo analiza un conjunto de condiciones del entorno que influyen en el desarrollo de Emprendimientos de Base Tecnológica (EBT) en economías basadas en innovación y en economías basadas en eficiencia. Se realiza una revisión de la literatura existente respecto a este fenómeno y se utilizan los datos del Global Entrepreneurship Monitor (GEM). Se lleva a cabo análisis factorial exploratorio con la aplicación del método de Rotación Ortogonal Varimax. El estudio concluye que algunos de los factores identificados resultan influyentes en determinados entornos económicos para la consolidación de los EBT's, particularmente en las economías innovadoras.

Palabras clave: Emprendimiento, base tecnológica, eficientes, innovadoras.

\section{Abstract}

This paper analyzes a set of environmental conditions that influence the development of Technology-Based Enterprises (TBCs) in innovation-based and efficiency-based economies. The existing literature on this phenomenon is reviewed and data from the Global Entrepreneurship Monitor (GEM) is used. Exploratory factorial analysis is handled out with the application of the Varimax Orthogonal Rotation method. The study concludes that some of the factors identified are influential in certain economic environments for the consolidation of EBT's, particularly in innovative economies.

Keywords: Entrepreneurship, technological base, efficient, innovative.

\footnotetext{
1 Universidad Técnica de Ambato. Facultad de Ciencias Administrativas. Marketing y Gestión de Negocios. Ambato-Ecuador. E-Mails: aaucanshala6059@uta.edu.ec, alexyz_77@hotmail.com.0RCID: https://orcid.org/0000-0002-9922-7058

2 Universidad Técnica de Ambato. Facultad de Ciencias Administrativas. Marketing y Gestión de Negocios. Ambato-Ecuador. E-Mails: otoainga8780@uta.edu.ec, oscarj_toainga@outlook.es. ORCID: https://orcid.org/0000-0002-4897-5144

3 Universidad Técnica de Ambato. Facultad de Ciencias Administrativas. Ambato - Ecuador. E-Mails: ramiropcarvajal@uta.edu.ec, patricio_56@yahoo.com. ORCID: https://orcid.org/0000-0003-1969-5490
} 


\section{Introducción}

El emprendimiento tiene un papel importante en el crecimiento de la economía global (Brunet \& Alarcón, 2004). Este término fue utilizado por primera vez en el año de 1755 por el economista Richard Castillón, la palabra emprendimiento tiene origen del francés entrepreneur, que significa pionero. Esta actividad se ha vuelto tendencia por su evidente aporte al desarrollo y dirección de nuevas empresas (Gutiérrez, 2018). El emprendimiento ha reinventado los sectores económicos de países y regiones clasificándose en varios tipos, por ejemplo: tradicional, por oportunidad, por necesidad, social, innovador, de base tecnológica, entre otros. Este último, ha tomado interés dentro de la sociedad por el uso de herramientas digitales, conocimiento e ingenio.

Los Emprendimientos de Base Tecnológica (EBT's) son definidos como iniciativas que se fundamentan en el desarrollo o combinación de tecnologías enfocadas a las necesidades del público objetivo (Torrecilla, Skotnicka \& Tous, 2017). Ciertas características denotan singularidad en estas empresas, como son: independencia, antigüedad menor a 25 años, inversión en I+D; capital humano competente; y su actividad principal es aprovechar los recursos tecnológicos disponibles (Zapata, López, Vivel, Neira \& Rodeiro, 2014). En comparación con los emprendimientos tradicionales los EBT's, generan productos con valor agregado utilizando pocos recursos, realizan alianzas con universidades e incubadoras para desarrollar actividades técnicas (Alarcón \& Díaz, 2016)..

El objetivo del artículo es analizar los factores del entorno que influyen en el emprendimiento de base tecnológica en economías basadas en eficiencia y economías basadas en innovación. Desde el punto de vista empírico se hace uso de la base de datos del Global Entrepreneurship Monitor (GEM) para el periodo 2008-2014, con el propósito de realizar un análisis factorial que permita identificar algunos de los factores principales que inciden sobre el fenómeno objeto de estudio.

Los EBT's han tenido un rol transcendental para la transformación y sostenibilidad de las economías. En este sentido, Maculan, Hernández \& Domínguez (2015) acotan que es el resultado de la revolución industrial que utiliza el conocimiento para automatizar procesos de producción, métodos y actividades. Asimismo, los EBT's pueden conformarse de manera individual o grupal para compartir conocimientos técnico-científicos y desarrollar productos o servicios con componentes tecnológicos (Aportela, 2015; García, García \& Olivares, 2018).

Díaz, Souto \& Tejeiro (2013) manifiestan que los EBT's son organizaciones que desarrollan productos y servicios en base a estudios previos y con mano de obra calificada. Éstas se forjan mediante la experiencia, manejo de herramientas digitales, conocimiento del mercado, habilidades individuales y colectivas (Alarcón \& Díaz, 2016). Este tipo de organizaciones utilizan otro enfoque de gestión estratégica, ubicación geográfica y mercado para formar su propio modelo de negocio (Zapata, Fernández \& Neira, 2018). Así también, los EBT's trabajan directamente con su segmento de clientes para recabar información, retroalimentarse, añadir valor, generar nuevas oportunidades de negocios y detectar necesidades insatisfechas (Cooper, 2006).

El éxito de los EBT's, viene definido primordialmente por los socios clave que estén dispuestos a proporcionar financiamiento, información del mercado y redes de negocio (Kirwan, Van der Sijde y Groen, 2006). Colovic y Lamotte (2015) mencionan que pese a tener limitaciones financieras, los EBT transforman información en conocimiento y lo usan para coordinar actividades, generar aplicaciones, automatizar procesos y elaborar productos novedosos para el mercado meta. En adición a esto, Alarcón \& Díaz (2016) y Zapata et al. (2018) sugieren que el éxito de una EBT está dado por el modelo productivo del entorno en el que se encuentra la empresa, puesto que éste debería estar orientado en la investigación, tecnología e innovación.

\section{Condiciones del entorno que influyen en el emprendimiento de base tecnológica}

En décadas recientes los EBT's han ganado presencia en el mercado, no obstante, éstos pueden ser influenciados de manera positiva 0 negativa por determinadas condiciones que afectan directa e indirectamente a este tipo de empresas. En las investigaciones de Borini, Ribeiro y Miranda (2017) los EBT's se enfrentan a desafíos de crecimiento constante; es decir, buscan ser más competitivos en el mercado global, caracterizado por la creciente innovación en productos/procesos. Por consiguiente, las economías han adoptado modelos productivos enfocados en investigación científica, innovación; y factores demográficos, económicos, políticos, recursos tangibles e intangibles para acelerar la constitución de EBT's (Barrientos, Quiroz \& López, 2017). En referencia a lo expresado en este apartado, se ha propuesto analizar las condiciones que se muestran a continuación.

\section{Gasto en I + D}

El gasto en I+D que realizan las organizaciones públicas y privadas contribuyen en el desarrollo del conocimiento tecnológico y creativo (Colovic \& Lamotte, 2015). Acs, Audretsch \& Lehmann (2013) afirman que sí el nivel de desarrollo de I+D en un país es alto, produce mayores conocimientos que son explotados por los empresarios 0 emprendedores, quienes desarrollan productos tecnológicos basados en la investigación. De lo contrario, sí el nivel de desarrollo es bajo, existe la posibilidad de que los emprendimientos tecnológicos sean adaptaciones de otras economías (Harbi \& Anderson, 2010). Por lo tanto, la cantidad de EBT en un país dependerá de la estructura de I+D en ese país.

Etemad (2016) indica que los gastos que implica la implementación de una estructura de I+D en una empresa naciente, es muy alto. Por lo que, los EBT's que empiezan su actividad económica, necesariamente deben buscar fuentes más accesibles a través de las universidades 0 
programas gubernamentales de investigación. Por otra parte, autores como Borini, Ribeiro \& Miranda (2017) mencionan que los EBT's que surgen en Economías Eficientes, en su mayoría, son imitaciones de Economías Innovadoras. Uno de los factores incidentes es la poca inversión en I+D de los países que conforman las Economías Eficientes.

\section{Educación}

La educación, desde sus inicios, debe incentivar en los individuos la actitud emprendedora de forma eficiente (Vicens \& Grullon, 2011). Con el propósito de que puedan evaluar sus capacidades y direccionarse hacia una idea de negocio. Ramaciotti, Muscio \& Rizzo (2017) mencionan que la educación y la experiencia laboral que se adquiere en alguna empresa del sector tecnológico, contribuyen en el desarrollo de nuevas capacidades emprendedoras direccionadas hacia la creación de EBT's.

Por su parte, Colovic \& Lamotte (2015) señalan que la educación superior aporta con el conocimiento para realizar un emprendimiento. De forma más específica, los conocimientos técnicos que se requieren para emprender en el sector tecnológico (Zapata et al., 2018). A diferencia de emprendimientos tradicionales, los de base tecnológica demandan de conocimientos técnicos especializados en un área. En compensación, las universidades y centros de capacitación producen especialistas competentes para diferentes áreas, además desarrollan investigaciones, cuyos resultados pueden ser difundidos de manera extensa y ser la base para el desarrollo de los productos de los EBT o para ser aprovechados por empresas de carácter tecnológico (Löfsten, 2016). Además, es importante que se generen redes con incubadoras, investigadores y otras empresas con más años de trayectoria en el mercado (Ramaciotti, Muscio \& Rizzo, 2017).

\section{Innovación}

Es evidente que la innovación ha reinventado el mercado de productos y servicios mediante la inclusión de herramientas tecnológicas en la gestión empresarial. Para Shane (2012) la innovación tecnológica consiste en experimentar en el mercado y generar cambios en el sector productivo. $Y$ para ello, se requiere de conocimientos técnicos, recursos, espíritu emprendedor y creatividad. Este tipo de innovación, ha sido diseñada netamente para los EBT's por contar con el ambiente económico adecuado y el capital humano requerido (Lima, 2016).

Los motivos para innovar por parte de los EBT's vienen dados por el conocimiento, manejo de Tecnologías de la Información y Comunicación (TICs), demanda del mercado, ciclo de vida del producto, entre otros (Lasso, Mainardes,\& Motoki, 2017). Añadiendo a esto, los autores An \& Ahn (2016) concuerdan que para innovar en este sector, las economías innovadoras trabajan basándose en el modelo de la tripe hélice: universidades (encargadas de proveer personal calificado); sector industrial (facilita el acceso a herramientas tecnológicas) y; el gobierno (establece políticas económicas y sociales que contribuyan al desarrollo de la innovación para las EBT).

\section{Financiamiento}

La estructura financiera de los EBT cambia conforme al tiempo de antigüedad en el mercado. Por lo general, inicia con capital propio o familiar y en cuanto empieza a crecer, accede a créditos comerciales 0 programas de financiamiento (Guercio, Martinez \& Vigier, 2017). Los recursos económicos se obtienen con la finalidad de perfeccionar los productos/servicios e incrementar las expectativas de crecimiento y pronto retorno de inversión (Bertoni, Martt \& Reverte, 2017). Sin embargo, las EBT tienen ciertos inconvenientes para acceder a financiamiento, estos pueden ser: falta de activos tangibles, el tiempo de maduración del producto/servicio y retorno de capital a largo plazo en comparación a empresas tradicionales (De los Ríos, Rodríguez \& Sáenz, 2015).

Dentro de esta perspectiva, resulta claro que, el acceso a crédito para estas empresas, no está dado por entidades financieras tradicionales. A criterio de Larsen, Vigier, Guercio \& Briozzo (2014) el tipo de financiamiento al que pueden acceder los EBT's son: capital semilla, capital emprendedor, o captar inversores ángel para fortalecer la competitividad en pequeñas y medianas empresas. De igual manera, ciertas economías proporcionan fondos de capital riesgo o programas gubernamentales para financiar emprendimientos de carácter tecnológico (Zea, Fonseca, Pérez, Bermeo \& Molina, 2016). Estos programas se encargan de realizar seguimiento periódico para garantizar el progreso y continuación de este tipo de empresas (Guercio et al., 2017).

\section{Incubadoras de Empresas de Base Tecnológica (IEBT)}

Las incubadoras constituyen organizaciones de apoyo y están dirigidas por entidades públicas o privadas que brindan recursos tangibles e intangibles, para acelerar el proceso de consolidar un EBT (Mian, Lamine \& Fayolle, 2016). Lasso et al. (2017) por su parte expresa que el objetivo de las incubadoras es transformar empresas incipientes en altamente competitivas con mayor oportunidad de éxito.

Ahora bien, a fin de mejorar el rendimiento de las incubadoras de base tecnológica, los autores Binsawad, Sohaib \& Hawryszkiewycz (2019) concuerdan que trabajar en convenio con universidades, atribuye beneficios como el acceso a mayor conocimiento en áreas técnicas y de gestión, así se reduce la probabilidad de fracaso, pero a la vez se extiende el tiempo de maduración de las empresas nacientes.

Por otro lado, Xiao \& North (2017) mencionan que las incubadoras deben trabajar directamente con las industrias de carácter tecnológico, de este modo, los EBT's pueden acceder a instalaciones y charlas con empresarios expertos en el mercado. La razón por la que aborda este pensamiento, se debe a que los EBT's tienen poca experiencia en lo que corresponde a la gestión de recursos y habilidades empresariales (McAdam \& Marlow, 2011). En 
consecuencia, los empresarios experimentados se encargan de instruir a emprendedores las estrategias para obtener financiamiento, inversores, maneras de direccionar empresas y formas de introducirse en el mercado (Zhang \& Sonobe, 2011)

\section{Políticas}

Tanto economías innovadoras como eficientes, han adoptado políticas gubernamentales para promover el conocimiento, creatividad, ciencia e innovación, con el fin de facilitar el acceso a herramientas e instituciones que proporcionen los recursos necesarios para constituir un EBT (Lecluyse, Knockaert \& Spithoven, 2018; Ramírez \& Fernández, 2018). Este tipo de políticas, buscan reformar el sistema educativo, promover la investigación nacional, incentivar el talento y facilitar el acceso al conocimiento mediante programas. Estos a su vez, ayudan a desarrollar tecnologías innovadoras que garantizan la trasformación de las economías (Aportela, 2015; Huang et al., 2013). Como resultado se obtienen centros de innovación, empresas basadas en ingeniería científica con alto grado de competitividad (Costantini \& Crespi, 2015).

Las políticas de gobierno son de vital importancia para la supervivencia de los EBT's. Generalmente surgen dos tipos de apoyo de las políticas gubernamentales: medidas fuertes, como capital semilla y préstamos con intereses mínimos; y medidas blandas como capacitaciones (Ramaciotti et al., 2017). En economías desarrolladas existen mejores oportunidades para la supervivencia de los EBTs. Un ejemplo es la estrategia de Lisboa y Europa 2020, que busca emprendimientos basados en los conocimientos tecnológicos, con crecimiento económico sostenible (Kuratko \& Menter, 2017).

\section{Metodología}

Para el estudio, se trabaja con información proporcionada por el Global Entrepreneurship Monitor (GEM), que es considerado como el estudio más importante sobre el emprendimiento en el mundo, puesto que analiza el panorama al que se enfrenta un emprendedor, su ecosistema, barreras sociales y contexto nacional. La información es recabada de la Encuesta Nacional a Expertos (NES) que cuenta con nueve Condiciones Marco Empresariales, de sus términos en inglés Entrepreneurial Framework Conditions (EFCs), que recogen datos consolidados y estandarizados correspondientes al contexto en el que se surgen los emprendimientos. La mencionada encuesta es aplicada a 36 expertos de cada país que conforma la muestra (College \& Park, 2017), lo cual valida la información con la que se ha trabajado.

Por su parte, el GEM agrupa a los países en tres tipos de economías: economías basadas en Innovación, en Eficiencia y en Factores Las primeras utilizan conocimientos y recursos tecnológicos para elaborar procesos/servicios más sofisticados que permiten generar valor. Las segundas se enfocan en los procesos de producción, mismos que se ven reflejados en la calidad y competitividad de sus productos/servicios. Sin embargo, las economías basadas en Factores en su mayoría son explotadoras de recursos primarios o productos de escaso valor añadido, además presentan procesos productivos ineficientes.

Al respecto, Vereinte Nationen (2018) manifiesta que la presencia de EBT's en las economías basadas en factores es escasa, razón por la cual se ha omitido el estudio de esta economía. En consecuencia, se decide trabajar con dos economías (innovadoras y eficientes). La muestra seleccionada para este estudio se conforma por 16 países; por un lado, ocho países para las economías innovadoras (Estados Unidos, Países Bajos, España, Italia, Suiza, Reino Unido, Alemania e Irlanda) y ocho países para las economías eficientes (Sudáfrica, Perú, Argentina, Brasil, Chile, Colombia, Croacia y Uruguay). Siendo el marco temporal el periodo comprendido entre 2008 a 2014 , selección que corresponde con la disponibilidad de información en la base de datos considerada.

En este trabajo se considera el Índice de Actividad Emprendedora (TEA) del sector tecnológico. Así como datos provenientes de seis de las nueve Condiciones Marco Empresariales (EFCs), que integra la información del NES (Tabla 1), puesto que tres de ellos no se relacionan con las actividades de carácter tecnológico, razón por la cual se excluyen de este análisis.

Tabla 1. Descripción de las Condiciones Marco Empresariales Condiciones

Marco Código Descripción

Empresariales

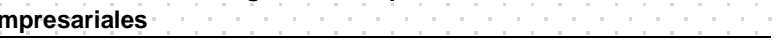

las variables de 5 puntos. Con
Financiamiento FIN apreciación de los expertos en el tema.

\section{$\mathrm{POL}$ variables de 5 puntos. Con apreciación de los expertos en el tema.}

\section{Incubadoras \\ (Programas} gubernamentales)

Resumen bloque C (INCUBADORAS PROGRAMAS GUBERNAMENTALES de las variables de 5 puntos. Con apreciación de los expertos en el tema.

Resumen bloque D1 (EDUCACIÓN EDU1 PRIMARIA Y SECUNDARIA) y D2 Educación (EDUCACIÓN SUPERIOR) de las variables de 5 puntos. Con apreciación EDU2 de los expertos en el tema.

Resumen bloque E (GASTO EN I+D) de las variables de 5 puntos. Con apreciación de los expertos en el tema.
INN1 Resumen bloque INN1 (INNOVACIÓN desde la perspectiva de las empresas) e INN2 (INNOVACIÓN desde la

Innovación perspectiva de los consumidores) de las

INN2 variables de 5 puntos. Con apreciación de los expertos en el tema.

Nota. La unidad de medida se presenta en promedio ponderado de la Escala de Likert ( 1 completamente falso, 5 completamente cierto).

Fuente: Elaboración propia a partir de la base de datos del GEM, en el periodo 2008-2014 
En la tabla 2 se muestran los estadísticos descriptivos de las economías analizadas, el cálculo del promedio en las economías innovadoras es de 2,858 , mismo que tiene una tendencia hacia la derecha (valor que se aproxima a 5 completamente verdadero según la escala de Likert), lo que explica que en estas economías los factores en estudio contribuyen significativamente en el desarrollo de las EBT's. A diferencia de las economías eficientes cuyo valor 2,608 representa un interés parcialmente por debajo de las economías innovadoras. En cuanto a la desviación estándar en la primera economía tiene un promedio de 0,348 de dispersión del punto medio, mientras que, la segunda economía posee un valor de 0,288 que representa una dispersión de datos más cercana en comparación a las economías innovadoras.

Tabla 2. Estadísticos descriptivos

\begin{tabular}{|c|c|c|c|c|c|}
\hline Economías & & Media & Mediana & $\begin{array}{l}\text { Desviación } \\
\text { estándar }\end{array}$ & Varianza \\
\hline \multirow{9}{*}{$\begin{array}{l}\text { Economía } \\
\text { Innovadoras }\end{array}$} & FIN & 2,638 & 2,69 & 0,364 & 0,133 \\
\hline & POL & 2,737 & 2,73 & 0,355 & 0,126 \\
\hline & INC & 2,957 & 3,05 & 0,457 & 0,209 \\
\hline & EDU1 & 2,104 & 2,07 & 0,387 & 0,15 \\
\hline & EDU2 & 2,845 & 2,83 & 0,34 & 0,116 \\
\hline & GID & 2,686 & 2,72 & 0,4 & 0,16 \\
\hline & INN1 & 3,285 & 3,31 & 0,281 & 0,079 \\
\hline & INN2 & 3,615 & 3,63 & 0,202 & 0,041 \\
\hline & Promedio & 2,858 & & 0,348 & \\
\hline \multirow{9}{*}{$\begin{array}{l}\text { Economía } \\
\text { Eficientes }\end{array}$} & FIN & 2,342 & 2,33 & 0,37 & 0,056 \\
\hline & POL & 2,425 & 2,35 & 0,376 & 0,141 \\
\hline & INC & 2,54 & 2,45 & 0,342 & 0,117 \\
\hline & EDU1 & 1,886 & 1,88 & 0,241 & 0,058 \\
\hline & EDU2 & 2,873 & 2,86 & 0,312 & 0,097 \\
\hline & GID & 2,24 & 2,18 & 0,251 & 0,063 \\
\hline & INN1 & 3,01 & 3,03 & 0,24 & 0,058 \\
\hline & INN2 & 3,546 & 3,57 & 0,175 & 0,031 \\
\hline & Promedio & 2,608 & & 0,288 & \\
\hline
\end{tabular}

Fuente: Elaboración propia a partir de la base de datos estadísticos

La tabla 3 presenta las correlaciones entre los datos generados, en el primer caso los datos tienen relaciones altas debido a que los coeficientes son mayores a 0,30 . Además, el valor de la determinante es 0,04 (valor próximo a cero) que permite inferir que las variables propias de la matriz, tienen un alto nivel de correlación lineal (Malhotra, 2008). Todo lo contrario, pasa con las Economías Eficientes que los datos muestran una débil correlación.
Tabla 3. Matriz de correlaciones

\begin{tabular}{cccccccccc} 
& & FIN & POL & INC & EDU1 & EDU2 & GID & INN1 & INN2 \\
\hline & FIN & 1 & 0,703 & 0,548 & 0,634 & 0,697 & 0,798 & 0,723 & 0,032 \\
& POL & 0,703 & 1 & 0,718 & 0,526 & 0,518 & 0,796 & 0,611 & 0,048 \\
& INC & 0,548 & 0,718 & 1 & 0,34 & 0,406 & 0,684 & 0,449 & $-0,311$ \\
$\begin{array}{c}\text { Economía } \\
\text { Innovadora }\end{array}$ & EDU1 & 0,634 & 0,526 & 0,34 & 1 & 0,797 & 0,625 & 0,631 & 0,239 \\
& EDU2 & 0,697 & 0,518 & 0,406 & 0,797 & 1 & 0,784 & 0,765 & 0,19 \\
& GID & 0,798 & 0,796 & 0,684 & 0,625 & 0,784 & 1 & 0,755 & 0,108 \\
& INN1 & 0,723 & 0,611 & 0,449 & 0,631 & 0,765 & 0,755 & 1 & 0,375 \\
& INN2 & 0,032 & 0,048 & $-0,311$ & 0,239 & 0,19 & 0,108 & 0,375 & 1 \\
\hline & FIN & 1 & 0,392 & $-0,226$ & $-0,225$ & $-0,608$ & $-0,321$ & 0,032 & $-0,123$ \\
& POL & 0,392 & 1 & 0,327 & 0,06 & $-0,101$ & $-0,038$ & 0,19 & $-0,034$ \\
& INC & $-0,226$ & 0,327 & 1 & 0,04 & 0,499 & 0,651 & 0,033 & $-0,068$ \\
& EDU1 & $-0,225$ & 0,06 & 0,04 & 1 & 0,555 & 0,113 & 0,141 & $-0,052$ \\
Economía \\
Eficiente
\end{tabular}

Como parte de la validación de datos se hace uso del Coeficiente de Alfa de Cronbach. Este método de validación presenta valores entre 0 y 1 , así los valores inferiores a 0,7 son considerados inaceptables y poco confiables para fines de investigación. Mientras que valores superiores a 0,7 revela una fuerte correlación entre el conjunto de datos. En la tabla 4 se pueden apreciar los coeficientes obtenidos para los datos de ambas economías, observándose que para la Economía Eficiente $(0,478)$ la condición antes mencionada no se cumple; por tanto, ésta se descarta del estudio y se procede con las pruebas estadísticas correspondientes para la Economía Innovadora $(0,898)$.

Tabla 4. Coeficiente de Alfa de Cronbach

\begin{tabular}{lcc} 
Economía & Alfa de Cronbach & N de elementos \\
\hline Economía Innovadora & 0,898 & 8 \\
Economía Eficientes & 0,478 & 8 \\
\hline
\end{tabular}

Fuente: Elaboración propia a partir de la base de datos del GEM, en el periodo 2008-2014

Asimismo, dada la naturaleza de los datos conjuntamente con la necesidad de identificar los factores latentes que se encuentran dentro de la matriz de correlaciones de las variables de estudio, se justifica el uso de la técnica estadística multivariante denominada Análisis Factorial Exploratorio (AFE). Adicionalmente, el número de datos para el estudio se encuentra en un rango de $300-400$ datos, lo cual permite trabajar con esta (Lloret, Ferreres, Hernández, \& Tomás, 2014).

A continuación, se realiza la matriz de componentes, que concentra la información de las variables originales, reduciéndolas a grupos más pequeños denominados variables residuales. Ésta por sí sola no proporciona una correcta interpretación de los factores; por lo que, se decide utilizar el método de Rotación Ortogonal Varimax, tratamiento que ayuda a identificar e interpretar de mejor 
manera las variables involucradas dentro de cada factor (Malhotra, 2008).

Por último, se presentan los resultados obtenidos y se procede a su interpretación a la luz del marco teórico y empírico analizado.

\section{Resultados}

Para confirmar que el modelo estadístico que se está aplicando es el adecuado, se realiza la prueba de esfericidad de Bartlett. Esto indica que mientras mayor sea su valor (Chi-cuadrado) y su nivel de significancia sea menor a 0,05 , se puede rechazar la hipótesis nula de que los factores mantienen correlación. Estos parámetros se evidencian en la tabla 5.

Tabla 5. Prueba de KMO y Bartlett para la economía innovadora

\begin{tabular}{|c|c|c|}
\hline \multicolumn{3}{|c|}{ Prueba de KMO y Bartlett } \\
\hline $\begin{array}{l}\text { Medida Kaiser-Meyer-Olk } \\
\text { muestreo }\end{array}$ & in de adecuación de & ,763 \\
\hline \multirow{3}{*}{$\begin{array}{l}\text { Prueba de esfericidad de } \\
\text { Bartlett }\end{array}$} & Aprox. Chi-cuadrado & 256,939 \\
\hline & gl & 28 \\
\hline & Sig. &, 000 \\
\hline
\end{tabular}

Fuente: Elaboración propia a partir de la base de datos estadísticos

La varianza total explicada determina el número de factores con los que se van a trabajar. En este estudio, la varianza total sugiere el uso de dos factores para la interpretación de datos, estas a su vez se detallan en la tabla 6.

Tabla 6. Varianza total explicada

\begin{tabular}{|c|c|c|c|c|c|c|c|c|c|}
\hline \multirow[b]{2}{*}{ Factoes } & \multicolumn{3}{|c|}{ Antavalores inirizhas } & \multicolumn{3}{|c|}{ 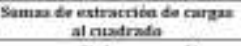 } & \multicolumn{3}{|c|}{$\begin{array}{l}\text { Simas de retartín de cargat al } \\
\text { euatrade }\end{array}$} \\
\hline & Texl & $\begin{array}{l}\text { Sode } \\
\text { marimes }\end{array}$ & 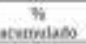 & Tokal & wote & sompisto & Totat & Thes & $\begin{array}{c}8 \\
\text { scumisede }\end{array}$ \\
\hline 1 & 4.912 & $61.5 \% 7$ & 61,397 & 4664 & $\sin 304$ & 50.304 & ง.มถ & $51 / A 0 t$ & 52601 \\
\hline 1 & 1.420 & 17,353 & 78,251 & toes & 12,562 & ro.s6s & 1.381 & 17,265 & 70856 \\
\hline 3 & 0,015 & 7091 & $a b, 862$ & & & & & & \\
\hline 4 & 0.345 & 4.306 & 91,148 & & & & & & \\
\hline 3 & 0,208 & 1,251 & 94,875 & & & & & & \\
\hline a & 0,105 & 2.436 & 97315 & & & & & & \\
\hline 7 & a,tut & Laot & 99,116 & & & & & & \\
\hline a & $\cos 1$ & .884 & 1000000 & & & & & & \\
\hline
\end{tabular}

Fuente: Elaboración propia a partir de la base de datos estadísticos

Una vez realizada la rotación por el método Varimax, se observa que el primer factor se conforma por: FIN, POL, INC, EDU1, EDU2, GID, INN1, Mientras que el segundo factor está compuesto por: EDU1, EDU2 y INN2, como se detalla en la tabla 7.

Tabla 7. Matriz Factorial Rotada

\begin{tabular}{|c|c|c|}
\hline & \multicolumn{2}{|c|}{ Factor } \\
\hline & 1 & 2 \\
\hline FIN & 0,803 & 259 \\
\hline POL & 0,855 &,- 031 \\
\hline INC & 0,830 &,- 342 \\
\hline EDU1 & 0,603 & ,526 \\
\hline EDU2 & 0,706 & ,566 \\
\hline GID & 0,919 & ,217 \\
\hline INN1 & 0,717 & ,474 \\
\hline INN2 & $-0,049$ &, 573 \\
\hline
\end{tabular}

Nota. Método de extracción: máxima verosimilitud. 2 factores extraídos. 7 iteraciones necesarias

Fuente: Elaboración propia a partir de la base de datos estadísticos
Los resultados muestran que el nivel de transferencia en I+D (GID) en el primer factor, tiene una carga factorial significativa, hecho que resulta coherente con lo expuesto por Acs, Audretsch \& Lehmann (2013) en su estudio, si el nivel de I+D de un país es alto, esto produce a mayor nivel de investigación e información que es utilizada por empresas 0 emprendedores para elaborar productos/servicios tecnológicos.

Del mismo modo, el Financiamiento (FIN) tiene un aporte considerable dado que, la naturaleza de los EBT conlleva riesgo por el grado de inversión e incertidumbre en su capacidad de payback (recuperación de inversión). Esto lo corrobora la investigación de Guercio, Martinez \& Vigier (2017) en la que sostienen que en economías innovadoras los EBT's prefieren acudir a financiamientos informales (inversores ángeles, mercados de capital, etc.) puesto que, mientras una empresa este más comprometida en desarrollar tecnología, disminuye la probabilidad de que recurran a financiamientos formales (entidades bancarias).

La valoración de la innovación desde el punto de vista de la empresa (INN1), presenta un valor moderado en el primer factor. Esto concuerda con los estudios de Sempere \& Hervás (2014) donde demuestra que en economías innovadoras, la innovación en emprendimientos o empresas relacionadas con tecnología, tiene mayor aporte siempre y cuando exista sinergia entre los recursos tecnológicos (herramientas digitales, acceso a centros especializados) y no tecnológicos (estructura organizacional, cultura, redes de contactos). Añadiendo a esto, los autores Zapata et al. (2018) sostienen que, existe mayor interés en apoyar a empresas o emprendimientos tecnológicos debido al grado de innovación que generan en el sector industrial.

En cuanto al aporte de las políticas gubernamentales (POL) en el factor uno, se aprecia que es positivo. Este valor es contrastado en las investigaciones de Cunningham, Lehmann, Menter \& Seitz (2019) donde sostienen que la adopción de políticas especializadas, muestran un efecto positivo en crear ecosistemas amigables tanto para emprendedores tradicionales como especialistas en tecnologías. Estas políticas se concentran en reformar la educación empresarial, financiar centros de innovación y facilitar el acceso a capital.

Respecto a la educación primaria y secundaria (EDU1) tiene un aporte relativamente significativo. No obstante, no tiene relación directa en la formación de emprendimientos de base tecnológica. Esto se menciona en el estudio de Brüne \& Lutz (2019) la educación empresarial a temprana edad incrementa la probabilidad de que los adolescentes participen en programas de emprendimiento en la universidad. Mientras que en la educación superior (EDU2) muestra un aporte significativo. Resultado que se corrobora con las investigación de Colovic \& Lamotte (2015) quienes indican que la educación superior contribuye en el desarrollo de los EBT's. Esto se debe a que, mientras mayor sea el grado de conocimiento técnico/científico de un emprendedor, es más probable que decida empezar un EBT (Zapata et al., 2018). 
En términos generales, en este estudio se pone de manifiesto la existencia de dos factores claramente identificados, por un lado, se encuentra el factor que recoge a elementos de las políticas públicas de un ecosistema innovador; $y$, por otro lado, un segundo factor que responde a aspectos que miden la educación y la innovación en los países seleccionados para el análisis.

\section{Conclusiones}

Desde la perspectiva de las condiciones del entorno abordadas en este estudio y que mantienen una estrecha relación con los EBT's, se comprueba que, en las Economías Innovadoras existe mayor concentración de los factores relacionados con financiamiento, políticas, incubadoras, gasto en I+D, innovación y educación. Los resultados obtenidos apuntan, a una influencia significativa de los mencionados factores sobre el fenómeno objeto de estudio; además, como se ha evidenciado en apartados anteriores, dicha influencia es coherente con lo señalado por otros estudios (Alarcón \& Díaz, 2016; Guercio et al., 2017; Zapata et al., 2014), lo que contribuye a confirmar que, en las Economías Innovadoras los EBT's cuentan con ecosistemas adecuados que permiten su gestación, crecimiento y sostenibilidad. Asimismo, los datos obtenidos podrían resultar de relevancia para la elaboración de diferentes políticas públicas en favor de la generación de EBT's.

Lo anterior se explica puesto que, en las economías examinadas, los EBT's han despertado especial interés por este tipo de empresas, por los efectos positivos en el desarrollo económico de los países. Por ende, se han efectivizado programas y políticas gubernamentales con el fin de contribuir en el desarrollo de dichos emprendimientos.

A pesar de que no se pudo llevar a cabo pruebas estadísticas contundentes con los datos referentes a las Economías Eficientes, queda claro entonces que las condiciones del entorno de dichas economías vienen determinadas por la carencia de políticas, herramientas tecnológicas, infraestructura o mano de obra calificada, que limitan su desarrollo económico en base a industrias de alta tecnología y se centran exclusivamente en la producción y explotación de recursos naturales y del sector turístico (Lima, 2016).

Además, en contraste con la revisión bibliográfica, la carencia de inversión en I+D en estas economías ocasiona que su desarrollo tecnológico sean imitaciones de las Economías Innovadoras. Asimismo, Zapata et al. (2016) en su investigación mencionan que no existe un efecto significativo de los EFCs sobre la Economía Eficiente. Por lo tanto, la poca representatividad justificaría el escaso desarrollo de los EBT's en los países que lo conforman.

En cualquier caso, los estudios llevados a cabo por Zapata \& Morales (2016); Zapata et al. (2018) señalan que existen diversos factores que influyen en diferente proporción para desarrollar EBT's en Economías Eficientes, entre los cuales se mencionan factores como: la edad del emprendedor tecnológico en un rango de 18 a 35 años puesto que los individuos están mejor adaptados al manejo de tecnologías.

Así también manifiestan que mientras mayor es el nivel de estudios, mayores son los conocimientos técnicocientíficos que ayudarán a familiarizarse con el sector tecnológico. Además, poseer una vida laboral activa y estar relacionado con otros emprendedores, generará experiencia y facilitará realizar gestiones administrativas. Por último, pero no por ello menos importante la ubicación geográfica del emprendedor y el aporte de las políticas para financiar este sector, parecen desempeñar un papel importante para la generación de este tipo de emprendimientos.

Las limitaciones no han estado ausentes en este estudio, tal como se ha podido apreciar en los aspectos metodológicos, ello ha obligado a centrar el estudio en un número reducido de países pertenecientes a un solo tipo de economía, con el objeto de obtener resultados concretos y ajustados a la realidad. En general, hubiese sido deseable disponer de información más robusta, lo cual contribuiría a captar mejor las relaciones de las condiciones del entorno de las economías eficientes. Se espera que en el futuro este tipo de carencias sean resueltas, proporcionando una mayor amplitud y variedad de información que contribuyan al avance del conocimiento sobre el fenómeno propuesto para el estudio.

En definitiva, el estudio exploratorio llevado a cabo constituye una primera evidencia de que determinados factores mantienen influencia sobre el fenómeno en estudio. En este caso particular, el primer factor identificado recoge aspectos relativos a políticas públicas del ecosistema innovador, mientras que el segundo factor está conformado por elementos relacionados con educación e innovación. Lo hallado en este artículo permite orientar posteriores investigaciones en la que se realicen análisis más robustos y confirmatorios de la influencia que tienen los mencionados factores sobre los emprendimientos de base tecnológica.

Por último, este estudio abre las posibilidades a su extensión y futuras líneas de investigación, como por ejemplo la posibilidad de extender el análisis a un estudio exclusivo de los factores relacionados con políticas públicas, educación e innovación, enfocados a la generación de EBT's en las economías innovadoras. Así mismo, no se debe olvidar que el fenómeno del emprendimiento de base tecnológica brota en entornos diversos y heterogéneos, con lo cual surge el interés de llevar a cabo un análisis de los factores que estarían incidiendo sobre los EBT's, particularmente, en economías eficientes.

\section{Agradecimientos}

A la Universidad Técnica de Ambato, Dirección de Investigación y Desarrollo (DIDE). Este artículo es producto del proyecto de investigación titulado "Estudio para la implementación del centro de desarrollo empresarial de la Facultad de Ciencias Administrativas", PFCA15. 


\section{Referencias}

Acs, Z. J., Audretsch, D. B., \& Lehmann, E. E. (2013). The knowledge spillover theory of entrepreneurship. Small Business Economics, 41(4), 757-774. https://doi.org/10.1007/s11187-013-9505-9

Alarcón, M. A., \& Díaz, C. del C. (2016). La empresa de base tecnológica y su contribución a la economía mexicana en el periodo 2004-2009. Contaduría y Administración, 61(1), 106-126. https://doi.org/10.1016/j.cya.2015.09.004

An, H. J., \& Ahn, S. J. (2016). Emerging technologiesbeyond the chasm: Assessing technological forecasting and its implication for innovation management in Korea. Technological Forecasting and Social Change, 102, 132142. https://doi.org/10.1016/j.techfore.2015.06.015

Aportela, I. (2015). La información como recurso estratégico en las empresas de base tecnológica. Revista General de Información y Documentación, 25(2). https://doi.org/10.5209/rev_RGID.2015.v25.n2.51238

Barrientos, E., Quiroz, L., \& López, W. (2017). Factores influyentes para el emprendimiento en tecnologías de la información y comunicación para economías de Latinoamérica vs economías de Europa y Norte América. Gestión de la innovación para la competitividad, 1-17.

Bertoni, F., Martt, J., \& Reverte, C. (2017). The Impact of Government-Supported Participative Loans on Employment Growth in Entrepreneurial Ventures. SSRN Electronic Journal. https://doi.org/10.2139/ssrn.3088072

Binsawad, M., Sohaib, O., \& Hawryszkiewycz, I. (2019). Factors impacting techology business incubator Performance. International Journal of Innovation Management, 23(01), 1950007. https://doi.org/10.1142/S1363919619500075

Borini, F., Ribeiro, F., \& Miranda, M. (2017). The internationalisation of new technology-based firms from emerging markets. 23-41.

Brüne, N., \& Lutz, E. (2019). The effect of entrepreneurship education in schools on entrepreneurial outcomes: A systematic review. Management Review Quarterly. https://doi.org/10.1007/s11301-019-00168-3

Brunet, I., \& Alarcón, A. (2004). Teorías sobre la figura del emprendedor. Papers. Revista de Sociologia, 73, 81. https://doi.org/10.5565/rev/papers/v73n0.1108

College, B., \& Park, B. (2017). Global Entrepreneurship Monitor report. 155.

Colovic, A., \& Lamotte, O. (2015). Technological Environment and Technology Entrepreneurship: A CrossCountry Analysis: Technological Environment and Technology Entrepreneurship. Creativity and Innovation
Management,

$24(4)$

https://doi.org/10.1111/caim.12133

617-628

Cooper, S. (2006). Exploring the pre-entrepreneurial careers of high-technology entrepreneurs. International Journal of Continuing Engineering Education and Life-Long Learning, 16(5), 341. https://doi.org/10.1504/IJCEELL.2006.010957

Costantini, V., \& Crespi, F. (2015). European enlargement policy, technological capabilities and sectoral export dynamics. The Journal of Technology Transfer, 40(1), 2569. https://doi.org/10.1007/s10961-013-9307-x

Cunningham, J. A., Lehmann, E. E., Menter, M., \& Seitz, N. (2019). The impact of university focused technology transfer policies on regional innovation and entrepreneurship. The Journal of Technology Transfer. https://doi.org/10.1007/s10961-019-09733-0

De los Ríos, S., Rodríguez, I., \& Sáenz, R. (2015). Emprendedores y capital riesgo en España: El caso de Fond-ICO Global. Revista Icade. Revista de las Facultades de Derecho y Ciencias Económicas y Empresariales, o(94), 31. https://doi.org/10.14422/icade.i94.y2015.002

Díaz, E., Souto, J., \& Tejeiro, M. (2013). Nuevas empresas de base tecnológica Netbiblo, S. L. https://www.madrimasd.org/uploads/informacionidi/bibliotec a/publicacion/doc/Nebts3.pdf

Etemad, H. (2016). Special Thematic Issue on: International Interactions and Activities of University-Based Technology Entrepreneurship. Journal of International Entrepreneurship, 14(3), 277-284. https://doi.org/10.1007/s10843-016-0189-5

García, A. M., García, M. G., \& Olivares, A. (2018). Entrepreneurs' Resources, Technology Strategy, and New Technology-Based Firms' Performance. Journal of Small Business Management. https://doi.org/10.1111/jsbm.12411

Guercio, M., Martinez, L., \& Vigier, H. (2017). Las limitaciones al financiamiento bancario de las Pymes de alta tecnología. Estudios Gerenciales, 33(142), 3-12. https://doi.org/10.1016/j.estger.2017.02.001

Gutiérrez Olvera, S. (2018). Emprendimiento en las empresas familiares / Entrepreneurship in family businesses. RICEA Revista Iberoamericana de Contaduría, Economía y Administración, 4(7), 163. https://doi.org/10.23913/ricea.v4i7.119

Harbi, S. E., \& Anderson, A. R. (2010). Institutions and the shaping of different forms of entrepreneurship. The Journal of Socio-Economics, 39(3), 436-444. https://doi.org/10.1016/j.socec.2010.02.011

Hernández, I., Álvarez, R., Blanco, C., \& Carvajal, A. (2014). El ascenso de la "mano invisible": Análisis para el surgimiento de un mercado formal de financiación para empresas de base tecnológica (ebt) en Colombia. FACE: 
Revista de la Facultad de Ciencias Económicas y Empresariales, 13(1), 5-32.

Huang, Y., Audretsch, D. B., \& Hewitt, M. (2013). Chinese technology transfer policy: The case of the national independent innovation demonstration zone of East Lake. The Journal of Technology Transfer, 38(6), 828-835. https://doi.org/10.1007/s10961-012-9292-5

harbiKirwan, P., Sijde, P., \& Groen, A. (2006). Assessing the needs of new technology-based firms (NTBFs): An investigation among spin-off companies from six European Universities. International Entrepreneurship and Management Journal, 2(2), 173-187. https://doi.org/10.1007/s11365-006-8683-1

Kuratko, D. F., \& Menter, M. (2017). The Role of Public Policy in Fostering Technology-Based Nascent Entrepreneurship. En J. A. Cunningham \& C. O'Kane (Eds.), Technology-Based Nascent Entrepreneurship (pp. 19-52). Palgrave Macmillan US. https://doi.org/10.1057/978-1-137-59594-2_2

Larsen, M., Vigier, H. P., Guercio, M. B., \& Briozzo, A. E. (2014). Financiamiento mediante obligaciones negociables. El problema de ser PyME. Visión de futuro, 18(2).

http://visiondefuturo.fce.unam.edu.ar/index.php/visiondefut uro/article/viewFile/26/18

Lasso, S., Mainardes, E., \& Motoki, F. (2017). Why do entrepreneurs open tech startups? A comparative study between Brazilian and foreign enterprises. International Entrepreneurship and Management Journal. https://doi.org/10.1007/s11365-017-0445-8

Lecluyse, L., Knockaert, M., \& Spithoven, A. (2018). The contribution of science parks: A literature review and future research agenda. The Journal of Technology Transfer. https://doi.org/10.1007/s10961-018-09712-x

Lima, R. (2016). Economic Growth and Human Capital in the Post-Knowledge Era: A Focus on Positive Externalities and Spillover Effects of Knowledge in Italy and the Emergency of the Less Developed Areas. Journal of Industrial Integration and Management, 01(03), 1650010. https://doi.org/10.1142/S242486221650010X

Lloret, S., Ferreres, A., Hernández, A., \& Tomás, I. (2014). El análisis factorial exploratorio de los ítems: Una guía práctica, revisada y actualizada. Anales de Psicología, 30(3), 1151-1169. https://doi.org/10.6018/analesps.30.3.199361

Löfsten, H. (2016). Business and innovation resources: Determinants for the survival of new technology-based firms. Management Decision, 54(1), 88-106. https://doi.org/10.1108/MD-04-2015-0139

Maculan, A.-M., Hernández, C. N. J., \& Domínguez, O. F. C. (2015). Aprendizaje en el proceso de incubación de empresas de base tecnológica. Económicas CUC, 36(1), 9-26. http://dx.doi.org/10.17981/econcuc.36.1.2015.21

Malhotra, N. K. (2008). Investigación de mercados. Pearson Educación.

McAdam, M., \& Marlow, S. (2011). Sense and sensibility: The role of business incubator client advisors in assisting high-technology entrepreneurs to make sense of investment readiness status. Entrepreneurship \& Regional Development, 23(7-8), 449-468. https://doi.org/10.1080/08985620903406749

Mian, S., Lamine, W., \& Fayolle, A. (2016). Technology Business Incubation: An overview of the state of knowledge. Technovation, 50-51, 1-12. https://doi.org/10.1016/j.technovation.2016.02.005

Ramaciotti, L., Muscio, A., \& Rizzo, U. (2017). The impact of hard and soft policy measures on new technology-based firms. Regional Studies, 51(4), 629-642. https://doi.org/10.1080/00343404.2016.1255319

Ramírez, M., \& Fernández, M. (2018). Unravelling the effects of Science Parks on the innovation performance of NTBFs. The Journal of Technology Transfer, 43(2), 482 505. https://doi.org/10.1007/s10961-017-9559-y

Sempere, F., \& Hervás, J. L. (2014). Innovación tecnológica y no tecnológica: Efectos complementarios en la performance empresarial. Economía Industrial, 2014, 71-76. https://riunet.upv.es/handle/10251/51948

Shane, S. (2012). Reflections on the 2010 AMD Decade Award: Delivering on the Promise of Entrepreneurship As a Field of Research. Academy of Management Review, 37(1), 10-20. https://doi.org/10.5465/amr.2011.0078

Torrecilla, J. A., Skotnicka, A. G., \& Tous, D. (2017a). Dimensiones que afectan a los emprendedores tecnológicos: El auge de las nuevas empresas de base tecnológica. 1-10. http://www.revistaespacios.com/a18v39n11/a18v39n11p16 .pdf

Vereinte Nationen (Ed.). (2018). Frontier technologies for sustainable development. United Nations.

Vicens, L., \& Grullon, S. (2011). Innovación y emprendimiento. Un modelo basado en el desarrollo del emprendedor. Banco Interamericano de Desarrollo, 1-31. https://publications.iadb.org/es/publicacion/15039/innovaci on-y-emprendimiento-un-modelo-basado-en-el-desarrollodel-emprendedor

Xiao, L., \& North, D. (2017). The graduation performance of technology business incubators in China's three tier cities: The role of incubator funding, technical support, and entrepreneurial mentoring. The Journal of Technology Transfer, 42(3), 615-634. https://doi.org/10.1007/s10961016-9493-4 
Zapata, G., López, S. F., Vivel, M., Neira, I., \& Rodeiro, D. (2014). El emprendimiento de base tecnológica; características diferenciales. 2-21. https://www.researchgate.net/publication/284545728_El_e mprendimiento_de_base_tecnologica_caracteristicas_difer enciales

Zapata, Á. R. P., \& Morales, L. I. R. (2016). Innovación y emprendimiento en América Latina Desafíos y oportunidades de la región para sumarse a la sociedad del conocimiento: México. 23.

Zapata, G., Fernández, S., \& Neira, I. (2018). EI emprendimiento tecnológico en Suramérica: Una aproximación a sus determinantes individuales. Perfiles Latinoamericanos, 26(52), 1-20. https://doi.org/10.18504/pl2652-003-2018

Zea, M. P. C., Fonseca, M. P., Pérez, R. A., Bermeo, M. R., \& Molina, X. C. (2016). Emprendimientos Informáticos: Una mirada desde la Universidad del Siglo XXI. Revista Didasc@ lia: Didáctica y Educación. ISSN 2224-2643, 7(3), 153-158.

Zhang, H., \& Sonobe, T. (2011). Business Incubators in China: An Inquiry into the Variables Associated with Incubatee Success. Economics: The Open-Access, OpenAssessment E-Journal, 5(2011-7), 1. https://doi.org/10.5018/economics-ejournal.ja.2011-7 